\title{
NICE ELONGATIONS OF PRIMARY ABELIAN GROUPS
}

\author{
Peter V. Danchev and Patrick W. Keef
}

\begin{abstract}
Suppose $N$ is a nice subgroup of the primary abelian group $G$ and $A=G / N$. The paper discusses various contexts in which $G$ satisfying some property implies that $A$ also satisfies the property, or visa versa, especially when $N$ is countable. For example, if $n$ is a positive integer, $G$ has length not exceeding $\omega_{1}$ and $N$ is countable, then $G$ is $n$-summable iff $A$ is $n$-summable. When $A$ is separable and $N$ is countable, we discuss the condition that any such $G$ decomposes into the direct sum of a countable and a separable group, and we show that it is undecidable in $\mathrm{ZFC}$ whether this condition implies that $A$ must be a direct sum of cyclics. We also relate these considerations to the study of nice bases for primary abelian groups.
\end{abstract}

\section{Introduction and Terminology}

By the term "group" we will mean an abelian $p$-group, where $p$ is a prime fixed for the duration. Our group theoretic terminology and notation will generally follow [11]. We will say a group $G$ is $\Sigma$-cyclic if it is isomorphic to a direct sum of cyclic groups. We will also utilize the language of valuated groups and valuated vector spaces (see [22] and [12], respectively); for example, if $Y$ is a valuated group, the letter $v$ will be reserved for its valuation, and for any ordinal $\alpha$, by $Y(\alpha)$ we will mean the subgroup $\{y \in Y: v(y) \geq \alpha\}$. We will implicitly assume that all valuated vector spaces are over $\mathbb{Z}_{p}$.

If $0 \rightarrow X \rightarrow G \rightarrow A \rightarrow 0$ is a short exact sequence, we will routinely identify $X$ with an actual subgroup of $G$ and $A$ with the quotient group $G / X$; we will say that $G$ is an elongation of $A$ by $X$. We then say that $G$ is a nice-elongation of $A$ if $X$ is a nice subgroup (i.e., for every $y \in G$, the $\operatorname{coset} y+X$ has an element of maximum height). Further,

2000 Mathematics Subject Classification. 20K10.

Key words. Nice subgroups, elongations, abelian groups, $\omega_{1}$-homomorphisms. 
if $X$ is countable, then we will say $G$ is an $\aleph_{0}$-elongation of $A$ by $X$. We can clearly combine these and speak of nice-\$ $\aleph_{0}$-elongations.

The main purpose of this paper is to investigate how certain properties of groups are preserved under nice- $\aleph_{0}$-elongations, though we will occasionally prove results that extend beyond the countable case. For example, if $\lambda \leq \omega_{1}$ is an ordinal, then a group $G$ is a $C_{\lambda}$-group if for every $\alpha<\lambda$, if $H$ is a $p^{\alpha}$-high subgroup of $G$ (i.e., $H$ is maximal with respect to the property that $H \cap p^{\alpha} G=\{0\}$ ), then $H$ is a dsc-group (i.e., a direct sum of countable groups). We verify that if $G$ is a nice-- $\aleph_{0}$-elongation of $A$, then $G$ is a $C_{\lambda}$-group iff $A$ is a $C_{\lambda}$-group (Corollary 2.4).

In another direction, a valuated group $Y$ will be said to be Honda if it is the ascending union of subgroups $X_{m}$ whose value spectra $\{v(x)$ : $\left.x \in X_{m}\right\}$ are finite (i.e., they are value-finite). Note that if $Y$ is actually countable, then it is clearly Honda, since it is the ascending union of finite subgroups.

If $G$ is a group with a subgroup $Y$, then restricting the height function on $G$ to $Y$ gives a valuation on $Y$. A group $G$ is summable if $G[p]$ is isometric to a free valuated vector space. More generally, following [8], if $n$ is a positive integer, a reduced group $G$ is $n$-summable if $G\left[p^{n}\right]$ is isometric to a valuated direct sum of countable valuated groups, and $G$ is $n$-Honda if $G\left[p^{n}\right]$ is Honda as a valuated group. In $[\mathbf{8}]$ it was shown that if $G$ is $n$-summable, then (a) it is summable, so that $p^{\omega_{1}} G=\{0\}$ (see, for example, [11, Theorem 84.3]); (b) the valuated group $G\left[p^{n}\right]$ is determined up to isometry by its Ulm function; (c) $G$ is $n$-Honda iff it is $n$-summable and has countable length (i.e., $p^{\alpha} G=\{0\}$ for some countable $\alpha$ ). In fact, these results were proven in the category of valuated $p^{n}$-socles (which extends the idea of a valuated vector space). Note that (c) generalizes the classical criterion (due to Honda) for the summability of a group of countable length (see, for example, [11, Theorem 84.1]).

If $G$ is a nice-elongation of $A$ by $N$, we show that if $N$ is countable and $G$ is $n$-summable, then $A$ is also $n$-summable (Theorem 1.1); and conversely, if $N$ is Honda (as a valuated group using the height valuation from $G), p^{\omega_{1}} G=\{0\}$ and $A$ is $n$-summable, then $G$ is $n$-summable (Theorem 1.2). It follows that if $G$ is a nice- $\aleph_{0}$-elongation of $A$ and $p^{\omega_{1}} G=\{0\}$, then $G$ is $n$-summable iff $A$ is $n$-summable (Corollary 1.3).

If $A$ is a separable group and $G$ is an elongation of $A$ by $X$, then $X$ will always be nice in $G$, so that an elongation of $A$ is always a niceelongation. Next, if $X=p^{\omega} G$ (so that $A \cong G / p^{\omega} G$ is separable), we will say that $G$ is an $\omega$-elongation of $A$ (this terminology agrees with $[\mathbf{2 0}]$ ), so that any $\omega$-elongation is also a nice-elongation; and if $X=p^{\omega} G$ is also countable, we will say $G$ is an $\omega-\aleph_{0}$-elongation of $A$. 
The group $G$ will be said to be countable plus separable (or cps for short), if it is isomorphic to $C \oplus S$, where $C$ is countable and $S$ is separable. Note that if $G=D \oplus R$, where $D$ is divisible and $R$ is reduced, then $G$ is cps iff $D$ is countable and $R$ is cps; we may occasionally, therefore, assume that some cps group is actually reduced. We will say that the separable group $A$ has the cps-elongation property if whenever the group $G$ is a (nice-) $\aleph_{0}$-elongation of $A$, then $G$ is cps; similarly we will say $A$ has the $\omega$-cps-elongation property if whenever the group $G$ is an $\omega$-ふ $\aleph_{0}$-elongation of $A$, then $G$ is cps. It is straightforward to show that any $\Sigma$-cyclic group has the cps-elongation property and that any group with the cps-elongation property has the $\omega$-cps-elongations property (Proposition 3.1); the natural question is whether a group with one of these latter two properties must actually be $\Sigma$-cyclic. We show that these questions are independent of the standard set-theoretic axioms (ZFC) by showing that they are consequences of the axiom of constructibility $(V=L)$, but that counter-examples can be constructed using Martin's Axiom and the denial of the Continuum Hypothesis (MA $+\neg \mathrm{CH}$, Theorem 3.4).

We then apply these results to question of when a group $G$ has a nice basis; that is, an ascending sequence of nice subgroups $X_{0} \subseteq X_{1} \subseteq$ $X_{2} \subseteq \cdots$ whose union is all of $G$ such that each $X_{m}$ is $\Sigma$-cyclic (see, for example, $[\mathbf{2}])$. In particular, we note that $\mathrm{MA}+\neg \mathrm{CH}$ implies that there is separable group $A$ which is not $\Sigma$-cyclic with the property that whenever $G$ is an $\omega$ - $\aleph_{0}$-elongation of $A$ by $X$, then $G$ has a nice basis (Example 4.7).

Referring to [6], a group homomorphism $\varphi: G \rightarrow A$ is said to be $\omega_{1}$-bijective if both the kernel and the co-kernel of $\varphi$ are countable. Thus, if there exists an $\omega_{1}$-bijective homomorphism $\varphi: G \rightarrow A$, then $G / \operatorname{ker} \varphi \cong$ $\varphi(G)$ and $G$ is an $\aleph_{0}$-elongation of $\varphi(G)$ by $\operatorname{ker} \varphi$. In particular, if $\operatorname{ker} \varphi$ is a countable nice subgroup of $G$ and $\varphi$ is surjective, then $G$ is a nice- $\aleph_{0}$-elongation of $A$; so there is a natural connection between these two concepts. We also establish some results pertaining to $\omega_{1}$-bijective homomorphisms of special classes of abelian groups (e.g., Proposition 3.6 and Theorem 5.6).

\section{1. n-Summable Groups}

A subgroup $X$ of a valuated group $Y$ is nice if every coset $y+X$ has an element of maximal value; such a $y$ is called proper with respect to $X$; and in this case, letting $v(y+X)=v(y)$ makes $Y / X$ into a valuated group. 


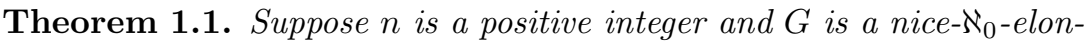
gation of $A$ by $N$. If $G$ is $n$-summable, then $A$ is $n$-summable.

Proof: Fix a valuated decomposition $G\left[p^{n}\right]=\oplus_{i \in I} C_{i}$, where each $C_{i}$ is a countable valuated group. We claim there is a countable subset $J \subseteq I$ such that:

(a) $N\left[p^{n}\right] \subseteq \oplus_{i \in J} C_{i}$;

(b) $\left(N+\oplus_{i \in J} C_{i}\right) \| G\left[p^{n}\right]$, that is, for every $x \in N+\oplus_{i \in J} C_{i}$ and $y \in$ $G\left[p^{n}\right]$ there is a $z \in\left(N+\oplus_{i \in J} C_{i}\right) \cap G\left[p^{n}\right]=\oplus_{i \in J} C_{i}$ such that $h t_{G}(x+z) \geq h t_{G}(x+y)$.

To begin, let $J_{0}$ be a countable subset of $I$ such that $N\left[p^{n}\right] \subseteq \oplus_{i \in J_{0}} C_{i}$. If we have constructed a countable subset $J_{k}$ of $I$, we want to construct another countable subset $J_{k+1}$ of $I$ containing $J_{k}$ such that:

(c) for every $x \in N+\oplus_{i \in J_{k}} C_{i}$ and $y \in G\left[p^{n}\right]$ there is a $z \in \oplus_{i \in J_{k+1}} C_{i}$ such that $h t_{G}(x+z) \geq h t_{G}(x+y)$.

For a second, fix $x$ to be an element of the countable subgroup $N+$ $\oplus_{i \in J_{k}} C_{i}$. We claim that there is countable sequence of elements $y_{x, \ell}$ of $G\left[p^{n}\right]$ such that

$$
\sup \left\{h t_{G}(x+y): y \in G\left[p^{n}\right]\right\}=\sup \left\{h t_{G}\left(x+y_{x, \ell}\right): \ell<\omega\right\} .
$$

This (essentially well-known) fact is a consequence of the assumption that $G\left[p^{n}\right]$ is a valuated direct sum of countable valuated groups and such an object will always be complete in the (induced) $\omega_{1}$-topology: To see this, note that if it failed, we could, for all $\alpha<\omega_{1}$, choose $y_{\alpha} \in G\left[p^{n}\right]$ such that $h t\left(x+y_{\alpha}\right) \geq \alpha$. It follows that if $\alpha<\alpha^{\prime}<\omega_{1}$, then $h t\left(y_{\alpha}-y_{\alpha^{\prime}}\right) \geq \alpha$. If for each $\alpha<\omega_{1}, y_{\alpha}=\left(z_{\alpha, i}\right)_{i \in I} \in \oplus_{i \in I} C_{i}$, then $z_{\alpha, i}$ must eventually be constant for each $i \in I$; say it takes on the value $w_{i}$. We next verify that all but finitely many of the $w_{i}$ must be 0 : If this failed, and $\omega_{1}>\alpha>h t\left(w_{i}\right)$ for an infinite number of indices $i$, it would easily follow that $z_{\alpha, i} \neq 0$ for this same infinite set of indices $i$, which cannot be. It follows that $w=\left(w_{i}\right)_{i \in I}$ is in $\oplus_{i \in J} C_{i}$, and that for all $\alpha<\omega_{1}, h t(x+w)=h t\left(x+y_{\alpha}+w-y_{\alpha}\right) \geq \alpha$, so that $x+w=0$; which means that we could let $y_{x, \ell}=w$ for all $\ell<\omega$.

We then let $J_{k+1}$ be a countable subset of $I$ containing $J_{k}$ such that $y_{x, \ell} \in \oplus_{i \in J_{k+1}} C_{i}$ for all $x \in N+\oplus_{i \in J_{k}} C_{i}$ and $\ell<\omega$.

If we then let $J=\cup_{k<\omega} J_{k}$, then (a) is immediate and (b) follows from (c).

Let $L=I-J$. If $x$ is a non-zero element of $\oplus_{i \in L} C_{i}$, we claim that $h t_{G}(x)=h t_{G / N}(x+N)$ : Note that if this failed, then since $N$ is nice in $G$, there would be an element $y \in N$ such that $h t_{G}(x+y)>$ $h t_{G}(x)$. Now, using (b), we could then find a $z \in \oplus_{i \in J} C_{i}$ such that 
$h t_{G}(x+z) \geq h t_{G}(x+y)>h t_{G}(x)$, but this contradicts the observation that $G\left[p^{n}\right]=\left(\oplus_{i \in J} C_{i}\right) \oplus\left(\oplus_{i \in L} C_{i}\right)$ is a valuated direct sum.

It follows that $\oplus_{i \in L} C_{i}=V$ embeds isometrically in $A\left[p^{n}\right]$. By Theorem 2.6 of $[\boldsymbol{8}]$, we will be done if we can show that $A\left[p^{n}\right] / V$ is countable. To that end, suppose $P$ is a countable pure subgroup of $G$ containing $N$. Note that if $z+N \in A\left[p^{n}\right]$, then $p^{n} z \in N \subseteq P$, so $p^{n} z=p^{n} w$ for some $w \in P$. Therefore, $z-w \in G\left[p^{n}\right]=\left(\oplus_{i \in J} C_{i}\right) \oplus V$, so $z-w=t+u$ where $t \in \oplus_{i \in J} C_{i}$ and $u \in V$. It follows that $z=(w+t)+u \in\left(P+\oplus_{i \in J} C_{i}\right)+V$, so that $A\left[p^{n}\right] \subseteq\left(P+\oplus_{i \in J} C_{i}\right) / N+V$, and since $\left(P+\oplus_{i \in J} C_{i}\right) / N$ is clearly countable, $A\left[p^{n}\right] / V$ must be countable, as well.

Recall that a countable valuated group is always Honda, so the following is essentially a converse of the above.

Theorem 1.2. Suppose $n$ is a positive integer, $G$ is a nice-elongation of $A$ by $N, p^{\omega_{1}} G=\{0\}$ and $N$ is Honda (as a valuated group using the height valuation from $G$ ). If $A$ is $n$-summable, then $G$ is $n$-summable.

Proof: Since the value spectrum of $N$ is countable, there is a countable ordinal $\lambda$ such that $N(\lambda)=N \cap p^{\lambda} G=\{0\}$. Let $G^{\prime}$ be a $p^{\lambda+n}$-high subgroup of $G$ containing $N$. Then $p^{\lambda} G^{\prime}$ is $p^{n}$-bounded and there is a decomposition $p^{\lambda} G=p^{\lambda} G^{\prime} \oplus X$ leading to an isometry $G\left[p^{n}\right]=G^{\prime}\left[p^{n}\right] \oplus$ $X\left[p^{n}\right]$, where the values in $X\left[p^{n}\right]$ are $\lambda$ plus the heights of elements computed in $X$ (cf. [8, Lemma 1.9]). Since $N(\lambda)=N \cap p^{\lambda} G=\{0\}$ and $N$ is nice, the map $p^{\lambda} G \rightarrow p^{\lambda} A$ is an isomorphism, so that $p^{\lambda} G$, and hence $X$, is $n$-summable. We therefore only need to show that $G^{\prime}$ is $n$-summable. Towards this end, note that $G^{\prime}$ is isotype in $G$ and $N$ is nice in $G^{\prime}$. It follows that $A^{\prime}=G^{\prime} / N$ embeds as an isotype subgroup of $A=G / N$. Since $p^{\lambda+n} A^{\prime}=p^{\lambda+n}\left(G^{\prime} / N\right)=\left[p^{\lambda+n} G^{\prime}+N\right] / N=\{0\}$, it follows that $A^{\prime}\left[p^{n}\right]$ is Honda. Without loss of generality, then, assume $G^{\prime}=G$ has countable length $\mu=\lambda+n$ and $G / N=A$ is $n$-Honda.

Let $M$ be the subgroup of $G$ containing $N$ satisfying $M / N=$ $(G / N)\left[p^{n}\right]=A\left[p^{n}\right]$. The height function on $G$ gives a valuation on $M$ and $M / N$ is the valuated direct sum $\oplus_{i \in I} V_{i}$, where $V_{i}$ is countable. For each coset in $M / N$, choose an element which is proper with respect to $N$ and let $M_{p} \subseteq M$ be the collection of all these proper elements.

Let $O_{j}$ for $j<\omega$ be an ascending sequence of finite sets of ordinals with union $\mu$.

For each $i \in I$, suppose $F_{i, j}$, for $j<\omega$ is an ascending chain of finite subgroups of $V_{i}$ whose union is all of $V_{i}$ such that the value spectrum 
of $F_{i, j}$ is contained in $O_{j}$. Let $F_{i, j}^{\prime}=\left\langle x \in M_{p}: x+N \in F_{i, j}\right\rangle$, so that $F_{i, j}^{\prime}$ is a finite group.

Suppose $N$ is the ascending union of the subgroups $X_{j}$ such that the value spectrum of $X_{j}$ is contained in $O_{j}$. Define

$$
Z_{j}=X_{j}+\left\langle F_{i, k}^{\prime}: i \in I, k \leq j \text { and } F_{i, k}^{\prime} \cap N \subseteq X_{j}\right\rangle .
$$

We claim that $M$ is the ascending union of these $Z_{j}$, and that the value spectrum of $Z_{j}$ is contained in $O_{j}$.

Claim 1. $Z_{j} \subseteq Z_{j+1}$.

First, note that $X_{j} \subseteq X_{j+1}$. Second, if $i \in I, k \leq j$, and $F_{i, k}^{\prime} \cap N \subseteq$ $X_{j}$, then $k \leq j+1$, and $F_{i, k}^{\prime} \cap N \subseteq X_{j} \subseteq X_{j+1}$, so that the claim follows.

Claim 2. $M=\cup_{j<\omega} Z_{j}$.

Note $N=\cup_{j<\omega} X_{j} \subseteq \cup_{j<\omega} Z_{j}$. Further, if $i \in I$ and $k<\omega$, then since $F_{i, k}^{\prime}$ is finite, we can find a $j \geq k$ such that $F_{i, k}^{\prime} \cap N \subseteq X_{j}$, and it follows that $F_{i, k}^{\prime} \subseteq Z_{j}$. It follows that $F_{i, k} \subseteq\left[\cup_{j<\omega} Z_{j}+N\right] / N=$ $\left[\cup_{j<\omega} Z_{j}\right] / N$, and since this happens for all $i$ and $k$, it follows that $M / N=\left[\cup_{j<\omega} Z_{j}\right] / N$, which implies the claim.

Claim 3. The value spectrum of $Z_{j}$ is contained in $O_{j}$.

Suppose

$$
z=x+g_{i_{1}}+\cdots+g_{i_{m}} \in Z_{j}
$$

where $x \in X_{j}$, and for $1 \leq \ell \leq m$, each $i_{\ell} \in I$ and for some $k_{\ell} \leq j$, $g_{i_{\ell}} \in F_{i_{\ell}, k_{\ell}}^{\prime}$, where $F_{i_{\ell}, k_{\ell}}^{\prime} \cap N \subseteq X_{j}$. Note that each $g_{i_{\ell}}$ is congruent modulo $F_{i_{\ell}, k_{\ell}}^{\prime} \cap N \subseteq X_{j}$ to some element of $F_{i_{\ell}, k_{\ell}}^{\prime} \cap M_{p}$, and so we actually assume each $g_{i_{\ell}}$ is proper with respect to $M$.

Note

$$
\begin{aligned}
v(z) & \leq v(z+N) \\
& =v\left(g_{i_{1}}+\cdots+g_{i_{m}}+N\right) \\
& =\min \left\{v\left(g_{i_{1}}+N\right), \ldots, v\left(g_{i_{m}}+N\right)\right\} \\
& =\min \left\{v\left(g_{i_{1}}\right), \ldots, v\left(g_{i_{m}}\right)\right\}
\end{aligned}
$$

which implies that

$$
v(x)=v\left(z-\left(g_{i_{1}}+\cdots+g_{i_{m}}\right)\right) \geq v(z),
$$

which further implies that

$$
v(z)=\min \left\{v(x), v\left(g_{i_{1}}\right), \ldots, v\left(g_{i_{m}}\right)\right\} \in O_{j} .
$$


It follows from Claims 2 and 3 that $M$ is Honda. We now observe that $G\left[p^{n}\right]$ is contained in $M$, and since $M$ is Honda, so is $G\left[p^{n}\right]$, so that $G$ must be $n$-summable, as required.

Since a countable valuated group is always Honda, the following is an immediate consequence of Theorems 1.1 and 1.2:

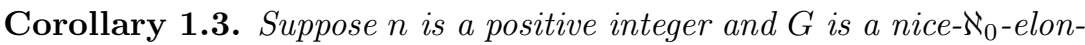
gation of $A$ with $p^{\omega_{1}} G=\{0\}$. Then $G$ is n-summable iff $A$ is $n$-summable.

Example 1.4. The hypothesis of niceness is necessary in Theorem 1.1.

As in Example 6.8 of [6], let $G_{0}$ be a group satisfying the following:

(a) $G_{0}$ is summable, $B$ is a countable unbounded $\Sigma$-cyclic group and there is an embedding of the torsion completion $\bar{B}$ in $G_{0}$ such that $p^{\omega} G_{0}=\bar{B}[p]$ and $G_{0} / \bar{B}$ is $\Sigma$-cyclic. [To construct such a $G_{0}$, let $H$ be a dsc-group of length $\omega+1$ such that there is a group isomorphism $\phi: p^{\omega} H \rightarrow \bar{B}[p]$, and let $G_{0}=[H \oplus \bar{B}] /\{(x, \phi(x))$ : $\left.x \in p^{\omega} H\right\}$, so $G_{0}$ is the sum of $H$ and $\bar{B}$ along $\phi$.]

Now, suppose $M$ is a countable group such that there is an isomor$\operatorname{phism} f: p^{\omega} M \rightarrow B$. Let $G=M \oplus G_{0}, X=\left\{(x, f(x)): x \in p^{\omega} M\right\} \subseteq G$, and $A=G / X$. It follows that

(b) $G$ is summable (since both $M$ and $G_{0}$ are summable);

(c) $X$ is countable (since $M$ is countable);

(d) $p^{\omega} A \cong \bar{B}$, so that $A$ is not summable.

To verify (d), note that clearly $B \cong B^{\prime}=[(\{0\} \oplus B)+X] / X \subseteq p^{\omega} A$, and since $A / B^{\prime} \cong(\bar{B} / B) \oplus\left(G_{0} / \bar{B}\right) \oplus\left(M / p^{\omega} M\right)$, where the first term of this decomposition is divisible and the last two terms are $\Sigma$-cyclic and hence separable, (d) then follows.

Example 1.5. Theorem 1.1 does not hold if the nice subgroup $N$ is only assumed to be Honda, as opposed to countable.

Suppose $A$ is any separable group which is not $\Sigma$-cyclic and $N \rightarrow G$ is a pure-projective resolution of $A$ (i.e., $G$ is $\Sigma$-cyclic and $N$ is pure in $G$ ), then $N$ is clearly Honda (since it is $\Sigma$-cyclic and the height valuation on $G$ and $N$ agree), $G$ is summable, but $A$ is not summable.

Example 1.6. The hypothesis $p^{\omega_{1}} G=\{0\}$ is necessary in Theorem 1.2. 
Suppose $G$ is a totally projective group with $N=p^{\omega_{1}} G$ countably infinite and $A=G / N$. Then $A$ is a dsc-group, and hence $A$ is summable. Since $p^{\omega_{1}} G \neq\{0\}$, however, $G$ will not be summable.

Example 1.7. The hypothesis of niceness is necessary in Theorem 1.2.

In Example 2.3 of [6], a separable group $G$ was constructed which was not $\Sigma$-cyclic but had a (pure) countable subgroup $X$ such that $A=G / X$ was a dsc-group of length $\omega+1$. It follows that this $A$ is summable, but $G$ is not; in fact $G$ is $p^{\omega+1}$-projective.

\section{Totally projective groups and generalizations}

A nice composition series for the valuated group $Y$ is an ascending chain of nice subgroups $\left\{X_{i}: i \leq \delta\right\}$ such that

(a) $X_{0}=\{0\}, X_{\delta}=Y$;

(b) for all $i<\delta, X_{i+1} / X_{i} \cong \mathbf{Z}_{p}$;

(c) for all limit ordinals $\lambda \leq \delta, X_{\lambda}=\cup_{i<\lambda} X_{i}$.

It is reasonably easy to verify that if $Y$ has a nice composition series, then so does $Y / Y(\alpha)$ for every ordinal $\alpha$, and that if $Y$ is Honda (so in particular, if $Y$ is countable), then it has a nice composition series. It is well known that a reduced group $G$ using the height valuation has a nice composition series iff it is totally projective (see, for example, [11, Theorem 81.9]). The following (essentially well-known) observation follows as a direct consequence.

Proposition 2.1. Suppose $G$ is a nice-elongation of $A$ by $N$. If $N$ has a nice composition series as a valuated group and $A$ is totally projective, then $G$ is totally projective.

Proof: If $\left\{X_{i}\right\}_{i \leq \delta}$ is a nice composition series for $N$, it can readily be checked that each $X_{i}$ is also nice in $G$. If $\left\{Z_{j}\right\}_{j<\epsilon}$ is a nice composition series for $A=G / N$, and for $j<\mu$ we let $Z_{j}^{\prime}$ be the subgroup of $G$ containing $N$ defined by the equation $Z_{j}^{\prime} / N=Z_{j}$, then each $Z_{j}^{\prime}$ will also be nice in $G$. It readily follows that $\left\{X_{i}\right\}_{i \leq \delta} \cup\left\{Z_{j}^{\prime}\right\}_{j \leq \epsilon}$ is a composition series for $G$, so $G$ is totally projective.

We include the following (also well-known) observation for future reference.

Corollary 2.2. Suppose $G$ is a reduced group which is a nice- $\aleph_{0}$-elongation of $A$ by $N$. Then $A$ is totally projective iff $G$ is totally projective. 
Proof: Sufficiency follows immediately from Proposition 2.1, and necessity follows directly from Proposition 1.1 of [6] (the second statement does not require the niceness of $N$ in $G$ ).

Recall that if $\lambda \leq \omega_{1}$, then $G$ is a $C_{\lambda}$-group if for every $\alpha<\lambda$, one (and so each) $p^{\alpha}$-high subgroup $H$ of $G$ is a dsc-group. If $\lambda$ is a limit, this is equivalent to requiring that $G / p^{\alpha} G$ is a dsc-group for all $\alpha<\lambda$, and if $\lambda$ is isolated, this is equivalent to requiring that one (and so each) $p^{\lambda-1}$-high subgroup $H$ of $G$ is a dsc-group (see, for example, the discussion in the first two paragraphs of Section 1 in [17]).

Theorem 2.3. Suppose $\lambda \leq \omega_{1}, G$ is a nice-elongation of $A$ by $N$ and $N$ has a nice composition series (using the height function on $G$ ). If $A$ is a $C_{\lambda}$-group then $G$ is a $C_{\lambda}$-group.

Proof: Assume $A$ is a $C_{\lambda}$-group. First, suppose $\lambda$ is a limit. Now, for all $\alpha<\lambda$ there is a nice short-exact sequence

$$
0 \rightarrow N / N(\alpha) \rightarrow G / p^{\alpha} G \rightarrow A / p^{\alpha} A \rightarrow 0 .
$$

Since $A / p^{\alpha} A$ is a dsc-group, it follows from Proposition 2.1 that $G / p^{\alpha} G$ must be a dsc-group. Since this holds for all $\alpha<\lambda$, it follows that $G$ is a $C_{\lambda}$-group, as required.

Next, suppose $\lambda=\gamma+1$ is isolated; so, in particular, $\lambda$ is countable. If $\lambda$ is finite, the result is trivial (since then any group is a $C_{\lambda}$-group), so suppose $\lambda$ is infinite. We therefore have a nice short-exact sequence:

$$
0 \rightarrow N / N(\lambda) \rightarrow G / p^{\lambda} G \rightarrow A / p^{\lambda} A \rightarrow 0 .
$$

Since a $p^{\gamma}$-high subgroup of $G$ maps to a $p^{\gamma}$-high subgroup of $G / p^{\lambda} G$, $G$ is a $C_{\lambda}$-group iff $G / p^{\lambda} G$ is a $C_{\lambda}$-group. Without loss of generality, then, replace $N, G$ and $A$ by $N / N(\lambda), G / p^{\lambda} G$ and $A / p^{\lambda} A$, so that we may assume all these groups have length at most $\lambda$.

Let $Y$ be a $p^{\gamma}$-high subgroup of $A$, then since $\gamma$ is infinite, we have that $A / Y$ is divisible and

$$
\left(p^{\gamma} A\right)[p]=p^{\gamma} A \rightarrow(A / Y)[p]
$$

is an isomorphism (see, for example, [14, Theorem 92]).

Let $X$ be the subgroup of $G$ containing $N$ such that $X / N=Y$. Note that

$$
G / X \cong(G / N) /(X / N)=A / Y
$$

is divisible and $\left(p^{\gamma} G\right)[p] \rightarrow(G / X)[p]$ can be factored as follows:

$$
\left(p^{\gamma} G\right)[p]=p^{\gamma} G \rightarrow p^{\gamma} A=\left(p^{\gamma} A\right)[p] \cong(A / Y)[p] \cong(G / X)[p]
$$


and since these maps are all surjective, it follows that $X$ is $p^{\lambda}$-pure in $G$ (see, for example, [14, Theorem 91]). This means that $X$ is isotype in $G$, so that

$$
0 \rightarrow N \rightarrow X \rightarrow Y \rightarrow 0
$$

is a nice sequence. Since $A$ is a $C_{\lambda^{-}}$-group, $Y$ is a dsc-group. By Proposition 2.1 this means that $X$ is also a dsc-group. Next, observe that $G[p]$ is isometric to a valuated direct sum $X[p] \oplus W$ where $W \subseteq p^{\gamma} G[p]$. This implies that if $X^{\prime}$ is a $p^{\gamma}$-high subgroup of $X$, then $X^{\prime}$ is also $p^{\gamma}$-high in $G$. Since $X^{\prime}$ will be isotype in $X$, it follows that $X^{\prime}$ is a dsc-group (see, for example, [14, Theorem 104]). However, this implies that $G$ is a $C_{\lambda}$-group, as required.

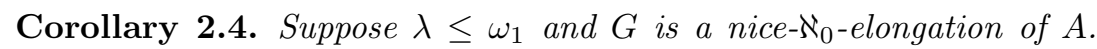

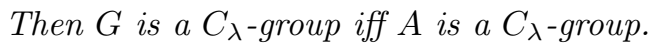

Proof: If $A$ is a $C_{\lambda}$-group, then Theorem 2.3 implies that $G$ is as well; and conversely, if $A$ is a $C_{\lambda}$-group, then Theorem 3.5 of [6] implies that $G$ is as well (the second implication does not require the niceness of $N$ in $G)$.

Remark 1. If $\lambda>\omega$, then Example 1.5 shows that in Corollary 2.4, if $N$ is only assumed to be Honda, as opposed to countable, then $A$ may be a $C_{\lambda}$-group, while $G$ is not.

For the remainder of this section $n$ will denote a fixed positive integer; $G$ is said to be an $n$ - $\Sigma$-group if $G\left[p^{n}\right]$ is the ascending union of a sequence of subgroups $X_{0} \subseteq X_{1} \subseteq X_{2} \subseteq \cdots$ such that for all $i<\omega, X_{i}(i)=$ $X_{i} \cap p^{i} G=\left(p^{\omega} G\right)\left[p^{n}\right]$. Utilizing Proposition 3.2 of [6], a group is an $n$ - $\Sigma$-group iff it is a $C_{\omega+n}$-group. Therefore, these last two results can be reformulated as follows:

Corollary 2.5. Suppose $G$ is a nice-elongation of $A$ by $N$.

(a) If $N$ has a nice composition series (using the height function on $G$ ) and $A$ is an $n$ - $\Sigma$-group, then $G$ is an $n-\Sigma$-group.

(b) If $N$ is countable, then $G$ is an $n$ - $\Sigma$-group iff $A$ is an $n$ - $\Sigma$-group.

A group $G$ is said to be pillared if $G / p^{\omega} G$ is $\Sigma$-cyclic. Since this is true iff for all $n<\omega, G / p^{\omega+n} G$ is a dsc-group, it follows that a group is pillared iff it is a $C_{\omega \cdot 2}$-group. Letting $\lambda=\omega \cdot 2$, then, we have the following: 
Corollary 2.6. Suppose $G$ is a nice-elongation of $A$ by $N$.

(a) If $N$ has a nice composition series (using the height function on $G$ ) and $A$ is pillared, then $G$ is pillared.

(b) If $N$ is countable, then $G$ is pillared iff $A$ is pillared.

A group $G$ is said to be $p^{\omega+n}$-projective if there is a subgroup $P \subseteq$ $G\left[p^{n}\right]$ such that $G / P$ is $\Sigma$-cyclic (e.g., $[21]$ ). By a classical result of Fuchs (see [13]), if $G_{1}$ and $G_{2}$ are $p^{\omega+n}$-projective, then $G_{1}$ and $G_{2}$ are isomorphic iff $G_{1}\left[p^{n}\right]$ and $G_{2}\left[p^{n}\right]$ are isometric. On the other hand if $P$ is any valuated group with $p^{n} P=\{0\}$ which is separable in the sense that $P(\omega)=\{0\}$, then there is a separable group $G$ containing $P$ such that the valuation on $P$ agrees with the height function on $G, G$ is separable and $G / P$ is $\Sigma$-cyclic; note that this $G$ will be $p^{\omega+n}$-projective.

More generally, imitating [3], a group $G$ is called $p^{\omega+n}$-totally projective if $p^{\omega} G$ is totally projective and $G / p^{\omega} G$ is $p^{\omega+n}$-projective. Clearly both $p^{\omega+n}$-projective groups and totally projective groups are themselves $p^{\omega+n}$-totally projective. By the same token, we define a group $G$ to be $p^{\omega+n}$-summable if $p^{\omega} G$ is summable and $G / p^{\omega} G$ is $p^{\omega+n}$-projective. Observe that $p^{\omega+n}$-projective groups are $p^{\omega+n}$-summable, whereas summable groups need not be $p^{\omega+n}$-summable since there exists a summable group with unbounded torsion-complete first Ulm factor and it is known that torsion-complete $p^{\omega+n}$-projective groups are bounded (see, for instance, $[\mathbf{1 6}])$.

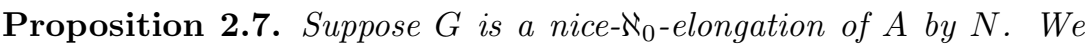
then have:

(a) $G$ is $p^{\omega+n}$-totally projective iff $A$ is $p^{\omega+n}$-totally projective.

(b) If $p^{\omega_{1}} G=\{0\}$, then $G$ is $p^{\omega+n}$-summable iff $A$ is $p^{\omega+n}$-summable.

Proof: Observe that $p^{\omega} G$ is a nice- $\aleph_{0}$-elongation of $p^{\omega} A$ by $N(\omega)=$ $N \cap p^{\omega} G$, and $G / p^{\omega} G$ is a nice- $\aleph_{0}$-elongation of $A / p^{\omega} A$ by $N / N(\omega)$. By Theorem 4.2 of [6], $G / p^{\omega} G$ is $p^{\omega+n}$-projective iff $A / p^{\omega} A$ is $p^{\omega+n}$-projective.

Therefore, (a) follows from Corollary 2.2 applied to $p^{\omega} G$ and $p^{\omega} A$, and (b) follows from Corollary 1.3 applied to the same.

\section{CPS groups and $\omega_{1}$-separable groups}

Proposition 3.1. Suppose $A$ is a separable group.

(a) If $A$ is $\Sigma$-cyclic, then it has the cps-elongation property.

(b) If $A$ has the cps-elongation property then it has the $\omega$-cps-elongation property. 
Proof: In (a), if $A$ is $\Sigma$-cyclic and $G$ is an $\aleph_{0}$-elongation of $A$ by $X$, then there is clearly a countable pure subgroup $Y$ of $G$ containing $X$ such that $Y / X$ is a summand of $A$. Since $G / Y \cong A /(Y / X)$ will be $\Sigma$-cyclic, it follows that $G \cong Y \oplus S$, for some $\Sigma$-cyclic group $S$, so that $G$ is cps. It follows that $A$ has the cps-elongation property.

The implication in (b) is obvious.

We now translate a notion that has been of considerable importance in the study of (torsion-free) free groups (see [10]) into the language of valuated vector spaces. We will say the valuated vector space $V$ is $\aleph_{1}$-coseparable if it is separable (i.e., $V(\omega)=\{0\}$ ), and for all subspaces $W$ of $V$ with $V / W$ countable there is a closed subspace $U$ of $V$ such that $U \subseteq W$ and $V / U$ is countable - note that this implies that the quotient valuated vector space $V / U$ is separable and hence free, so that $V$ is isometric to $U \oplus F$ where $F$ is countable and free. Note also that if $V / W$ is unbounded (i.e., $(V / W)(m) \neq\{0\}$ for all $m<\omega$ ), then the same will be true of $F$.

We pause to review the following standard construction (see, for example, $[\mathbf{1 4}$, Theorem 106]).

Lemma 3.2. Suppose $A$ is a separable group, $D \subseteq A[p]$ is a dense subsocle and $X$ is a group such that $A[p] / D \cong X / p X$. Then there is an $\omega$-elongation $G$ of $A$ by $X$ such that $D=\left[G[p]+p^{\omega} G\right] / p^{\omega} G \subseteq A[p]$.

Proof: Let $Y$ be a pure and dense subgroup of $A$ such that $Y[p]=D$, so that $A / Y$ is divisible; and let $Z$ be a divisible hull for $X$, so that $Z / N$ is also divisible. The isomorphism $A[p] / D \cong X / p X$ implies that there is an isomorphism $\phi: A / Y \rightarrow Z / X$, and we can let $G=\{(a, z) \in A \oplus Z$ : $f(a+Y)=z+X\}$. The assignment $x \rightarrow(0, x)$ gives an injection $X \rightarrow G$, and the assignment $(a, z) \rightarrow a$ gives a surjection $G \rightarrow A$, and it can be checked that these conditions will imply the conclusions.

Theorem 3.3. The following conditions are equivalent (in ZFC):

(a) Every separable group $A$ with the $\omega$-cps-elongation property is $\Sigma$ cyclic.

(b) Every separable $p^{\omega+1}$-projective group A with the $\omega$-cps-elongation property is $\Sigma$-cyclic.

(c) Every $\aleph_{1}$-coseparable valuated vector space is free (as a valuated vector space).

Proof: It is clear that (a) implies (b). Suppose next that (b) holds and that $V$ is $\aleph_{1}$-coseparable; we want to show that $V$ must be free. Let $A$ be a separable group containing $V$ as a subgroup where $A / V$ is $\Sigma$-cyclic, 
so that $A$ is $p^{\omega+1}$-projective. Since there is a valuated injection of the valuated quotient space $A[p] / V$ into $(A / V)[p]$ and the latter is Honda, so is $A[p] / V$, and hence free as a valuated vector space. It follows that $A[p]$ is isometric to $V \oplus F$ where $F$ is a free valuated vector space. In fact, after possibly adding to $A$ a summand which is an infinite $\Sigma$-cyclic group, we may assume that every Ulm invariant of $F$ is infinite.

We now show that this $A$ has the $\omega$-cps-elongation property. To this end, let $G$ be an $\omega$-^ $\aleph_{0}$-elongation of $A$, so that $A=G / p^{\omega} G$; we need to show $G$ is cps. We begin by letting $W^{\prime}=\left[G[p]+p^{\omega} G\right] / p^{\omega} G \subseteq$ $A[p]$. It can easily be checked that $\phi\left(x+p^{\omega} G\right)=p x+p^{\omega+1} G$ gives a well-defined homomorphism $A[p] \rightarrow p^{\omega} G / p^{\omega+1} G$ whose kernel is $W^{\prime}$. It follows that $A[p] / W^{\prime}$ embeds in the countable group $p^{\omega} G / p^{\omega+1} G$, and hence $A[p] / W^{\prime}$ is also countable.

Now let $W=W^{\prime} \cap V$. Since $V / W$ embeds in $A[p] / W^{\prime}$, it follows that $V / W$ is countable. Let $U$ be a closed subspace of $V$ contained in $W$ such that $V / U$ is countable. Finally, let $P$ be a subgroup of $G[p]$ such that $G[p] \rightarrow W^{\prime}$ maps $P$ isomorphically onto $U$; since the homomorphism $G[p] \rightarrow W^{\prime} \subseteq A[p]$ preserves all finite heights, it follows that $P$ also maps isometrically onto $U$.

From this construction we can conclude that $\left[p^{\omega} G \oplus P\right] / p^{\omega} G=U$. Finally, let $Q=p^{\omega} G \oplus P \subseteq G$, so that $G / Q \cong\left(G / p^{\omega} G\right) /\left(\left[p^{\omega} G \oplus\right.\right.$ $\left.P] / p^{\omega} G\right)=A / U$.

Claim. The decomposition $Q=p^{\omega} G \oplus P \subseteq G$ is valuated, $G / Q$ is $\Sigma$-cyclic and, for all $m<\omega$, the relative Ulm function $f_{G, Q}(m)$ is infinite.

Since $p^{\omega} G \cap P=\{0\}$, the first statement readily follows. Since $G / Q \cong$ $A / U$, we must show that the latter is $\Sigma$-cyclic. Observe that $V / U$ is a free, separable valuated vector space, so there is a valuated embedding $V / U \rightarrow J$, where $J$ is a $\Sigma$-cyclic group using the height valuation. Since $V$ is nice in $A$ and $A / V$ is $\Sigma$-cyclic, it follows that this embedding extends to a homomorphism $f: A \rightarrow J$, for which the kernel of $\left.f\right|_{V}$ is $U$. It follows that the kernel of the obvious map $A \rightarrow(A / V) \oplus J$ is $U$, and since $(A / V) \oplus J$ is $\Sigma$-cyclic, so is $A / U$.

Observe that if $m<\omega$, then $f_{G, Q}(m)=f_{A, U}(m)$. Since $F$ has infinite Ulm invariants, $U \subseteq V$, and $A[p]$ is isometric to $V \oplus F$, the claim follows.

Let $C$ be a countable group whose Ulm function is defined by

$$
f_{C}(\alpha)= \begin{cases}f_{G}(\alpha), & \text { when } \omega \leq \alpha \leq \infty \\ 1, & \text { when } \alpha<\omega\end{cases}
$$


Note this implies that $p^{\omega} C$ and $p^{\omega} G$ are isomorphic, even when they are not reduced. Let $H=C \oplus A$, so that $Q^{\prime}=p^{\omega} C \oplus U$ is nice in $H$, and $H / Q^{\prime} \cong\left(C / p^{\omega} C\right) \oplus(A / U)$ is $\Sigma$-cyclic. In addition, $\mathrm{Q}^{\prime}$ and $\mathrm{Q}$ are both isometric to $p^{\omega} G \oplus U$ and so they are isometric to each other. Next, it is readily checked that if $\alpha \geq \omega, f_{G, Q}(\alpha)=0=f_{H, Q^{\prime}}(\alpha)$ and if $m<\omega$,

$$
f_{H, Q^{\prime}}(m)=f_{G, Q}(m)+1=f_{G, Q}(m) .
$$

Therefore, by the fundamental result of Hill on extending isometries on nice valuated subgroups of groups (see, for example, [11, Theorem 83.4]), it follows that there is an isomorphism $G \rightarrow H=C \oplus A$. It therefore follows that $G$ is cps, so that $A$ has the $\omega$-cps-elongation property. By hypothesis, then, $A$ must be $\Sigma$-cyclic, and this implies that $A[p]$ is free as a valuated vector space, and since $V$ is a subspace of $A[p]$, it is also free, as required.

We now need to show that (c) implies (a), so suppose every $\aleph_{1}$-coseparable valuated vector space is free and $A$ is a separable group with the $\omega$-cps-elongation property, and let $V=A[p]$; it follows trivially that $V$ is separable. Let $W$ be a subspace of $V$ such that $V / W$ is countable. Note that if $\bar{W}$ is the $p$-adic closure of $W$ in $V$, then $\bar{W} / W=(V / W)(\omega)$. Since $V / W$ is countable, there is a subspace $W^{\prime}$ of $V$ containing $W$ such that $V / W$ is isometric to the valuated direct sum $\left(W^{\prime} / W\right) \oplus(\bar{W} / W)$. Note that $W$ is closed (and hence, nice) in $W^{\prime}$ and $W^{\prime} / W$ is countable (and hence, free), so that $W^{\prime}=W \oplus F$ for some countable, free subspace $F$.

Note that $W^{\prime}$ is dense in $V$. By Lemma 3.2 there is an $\omega$-elongation $G$ of $A$ such that $p^{\omega} G$ is countable, $G / p^{\omega} G=A$ and $\left[G[p]+p^{\omega} G\right] / p^{\omega} G=W^{\prime}$.

By hypothesis, $G=C \oplus S$, where $C$ is countable and $S$ is separable. First, note that we can identify $A[p]=V$ with $\left(C / p^{\omega} C\right)[p] \oplus S[p]$, so that we can view $S[p]$ as a subgroup of $W^{\prime} \subseteq A[p]=V$. We now let $U=S[p] \cap W$. Since both $V / S[p]$ and $V / W$ are countable, and $V / U$ embeds in $(V / S[p]) \oplus(V / W)$, it follows that $V / U$ is countable.

To show that $U$ is closed, let $u_{i}$ for $i<\omega$ be a sequence in $U$ which converges in the $p$-adic topology to $z \in V$. Since $A[p]=\left(C / p^{\omega} C\right)[p] \oplus$ $S[p], S[p]$ is closed in $V$, so that $z \in S[p] \subseteq W^{\prime}$. In the decomposition $W^{\prime}=W \oplus F$, each $u_{i} \in W$, so that $z \in W$, as well. It follows that $z \in S[p] \cap W=U$.

Note that the arguments in the last two paragraphs imply that $V$ is $\aleph_{1}$-coseparable. It follows, therefore, that $V=A[p]$ is free, so that $A$ is $\Sigma$-cyclic, as required. 
Theorem 3.4. The following hold:

(a) Assuming $(M A+\neg C H)$, there is a separable group $A$ which has the cps-elongation property (and hence it also has the $\omega$-cps-elongation property), but is not $\Sigma$-cyclic.

(b) Assuming $(V=L)$, if $A$ is a separable group which has the $\omega$-cpselongation property (in particular, if $A$ has the cps-elongation property), then $A$ is $\Sigma$-cyclic.

Before proceeding, note that the last result implies the following.

Corollary 3.5. The following statements are independent of ZFC:

(a) Every separable group with the cps-elongation property is $\Sigma$-cyclic.

(b) Every separable group with the $\omega$-cps-elongation property is $\Sigma$ cyclic.

(c) Every $\aleph_{1}$-coseparable valuated vector space is free.

Proof: By Theorem 3.4, both (a) and (b) fail in a model of $\mathrm{MA}+\neg \mathrm{CH}$ and both are true in $V=L$. By Theorem 3.3, (b) is equivalent to (c).

Recall that a group $A$ is $\omega_{1}$-separable iff it is separable and every countable subgroup of $A$ is contained in a countable summand of $A$. Using a (by now) standard construction, it can be shown in ZFC that there are $\omega_{1}$-separable groups of cardinality $\aleph_{1}$ which are not $\Sigma$-cyclic.

Proof of Theorem 3.4: Regarding (a), suppose $A$ is an $\omega_{1}$-separable group of cardinality $\aleph_{1}$ which is not $\Sigma$-cyclic. If $G$ is an $\aleph_{0}$-elongation of $A$ by $X$, then let $P$ be a countable pure subgroup of $G$ containing $X$. Since $A=G / X$ is $\omega_{1}$-separable, we can find a countable subgroup $C$ of $G$ containing $P$ such that $C / X$ is a summand of $G / X=A$.

We claim that $C$ will be pure in $G$ : Note that $C / X$ is clearly pure in $G / X$, which implies that $C / P \cong(C / X) /(P / X)$ is pure in $G / P \cong$ $(G / X) /(P / X)$. Since $P$ is pure in $G$, it follows that $C$ is pure in $G$, as required.

If we let $A=A^{\prime} \oplus(C / X)$, then the countability of $C / X$ and the fact that $A$ is $\omega_{1}$-separable readily implies that $A^{\prime}$ is also $\omega_{1}$-separable. In the presence of $\mathrm{MA}+\neg \mathrm{CH}$, by Theorem 3.1 of $[\mathbf{1 8}]$, we can conclude that $\operatorname{Pext}\left(A^{\prime}, C\right)=\{0\}$. However, since $C$ is pure in $G$ and $G / C \cong$ $(G / X) /(C / X) \cong A^{\prime}$, we can conclude that $G \cong A^{\prime} \oplus C$, as required.

Regarding (b), note that if $G$ is an $\omega$-elongation of $A$ by $\mathbb{Z}_{p}$, then since $A$ has the $\omega$-cps-elongation property, it follows that $G \cong C \oplus S$, where $C$ is countable and $S$ is separable. If $H_{\omega+1}$ is the generalized Prüfer group of length $\omega+1$, there is clearly a homomorphism $G \rightarrow C \rightarrow H_{\omega+1}$ which 
is non-zero on $p^{\omega} G=p^{\omega} C$. In the presence of $V=L$, by Theorem 2.2 of [20], $A$ must be $\Sigma$-cyclic.

Although by Proposition 7.2 of [4], $A$ being $\omega_{1}$-separable implies that $A / C$ is $\omega_{1}$-separable whenever $C$ is a countable nice subgroup of $A$, we shall see that the converse implication does not hold in some versions of set theory. First, we pause for the following assertion which answers Problem 7.3 from [4].

Proposition 3.6. Suppose $A$ is a separable group and $f: G \rightarrow A$ is an $\omega_{1}$-bijective homomorphism. If $G$ is $\omega_{1}$-separable, then $A$ is $\omega_{1}$-separable.

Proof: Let $I$ be the image of $f$ and $K$ be the kernel of $f$. Since $I$ is a subgroup of $A, K$ is a countable and nice subgroup of $G$, so by Proposition 7.2 of $[4], I \cong G / K$ is $\omega_{1}$-separable. Now, if $C$ is a countable subgroup of $A$, then after possibly expanding $C$, we may assume that $A=I+C$. Let $C^{\prime}$ be a countable subgroup of $I$ containing $I \cap C$ such that $I=I^{\prime} \oplus C^{\prime}$; the existence of such a $C^{\prime}$ follows from the fact that $I$ is $\omega_{1}$-separable. Then $C \subseteq C+C^{\prime}$ is countable and one easily checks that $A=I^{\prime} \oplus\left(C^{\prime}+C\right)$. It follows that $A$ is $\omega_{1}$-separable, as desired.

The next result shows that the converse of Proposition 3.6 is undecidable in ZFC for groups of cardinality $\aleph_{1}$. It settles Problem 7.2 from [4].

Theorem 3.7. (a) $(M A+\neg C H)$ If the separable group $G$ is an $\aleph_{0}$ elongation of the $\omega_{1}$-separable group $A$ and $|A|=\aleph_{1}$, then $G$ is also $\omega_{1}$-separable.

(b) $(V=L)$ There is a separable group $G$ which is an $\aleph_{0}$-elongation of an $\omega_{1}$-separable group $A$ with $|A|=\aleph_{1}$ such that $G$ is not $\omega_{1}$ separable.

Proof: Regarding (a), a separable group $H$ is weakly $\omega_{1}$-separable if for every countable subgroup $C$ of $H$, the $p$-adic closure $\bar{C}$ is also countable. By [19], in the presence of MA $+\neg \mathrm{CH}$, for groups of cardinality $\aleph_{1}$, the classes of weakly $\omega_{1}$-separable and $\omega_{1}$-separable groups coincide. Finally, in view of Corollary 5.2 of $[\mathbf{6}]$ we have that $G$ is weakly $\omega_{1}$-separable iff $A$ is weakly $\omega_{1}$-separable.

Regarding (b), Megibben [19, Theorem 3.2] found in the presence of $V=L$ an $\omega_{1}$-separable group $A$ of cardinality $\aleph_{1}$ containing a pure and dense subgroup $G^{\prime}$ which is not $\omega_{1}$-separable such that $A / G^{\prime}$ is countable. If $C$ is a countable subgroup of $A$ such that $G^{\prime}+C=A$, then let $G=$ $G^{\prime} \oplus C$ (note this is an external direct sum). Since $G^{\prime}$ is not $\omega_{1}$-separable, the countability of $C$ easily implies that $G$ is not $\omega_{1}$-separable. If we 
then consider the sum map $G=G^{\prime} \oplus C \rightarrow G^{\prime}+C=A$, defined by $(g, c) \mapsto g+c$, we have our result.

\section{Groups with nice bases}

Following [2], recall that a nice basis for a group $G$ is an ascending sequence of nice subgroups $X_{0} \subseteq X_{1} \subseteq X_{2} \subseteq \cdots$ whose union is all of $G$ such that each $X_{m}$ is $\Sigma$-cyclic.

Proposition 4.1. Let $G$ be a nice- $\aleph_{0}$-elongation of $A$ by $N$ such that $N \cap p^{\omega} G=\{0\}$. If $A$ has a nice basis, then $G$ has a nice basis.

Proof: Write $G / N=\cup_{i<\omega}\left(G_{i} / N\right)=\left(\cup_{i<\omega} G_{i}\right) / N$, where $N \leq G_{i} \subseteq$ $G_{i+1} \leq G$ and all $G_{i} / N$ are nice in $G / N$ and are $\Sigma$-cyclic groups. Therefore, $G=\cup_{i<\omega} G_{i}$ with $p^{\omega} G_{i} \subseteq N$. Hence $p^{\omega} G_{i} \subseteq N \cap p^{\omega} G=\{0\}$ and we conclude that all $G_{i}$ are separable. By Corollary 2.2, they are $\Sigma$-cyclic groups. Moreover, utilizing Lemma 79.3 of [11], we derive that these $G_{i}$ are also nice in $G$, as needed.

The last result can also be deduced from [4, Proposition 9.1]; nevertheless we have given another, more smooth, proof.

Example 4.2. In Proposition 4.1, the restriction $N(\omega)=N \cap p^{\omega} G=\{0\}$ is necessary.

Suppose $A$ is any separable thick group and let $G$ be any group such that $p^{\omega} G$ is countable, reduced inseparable (thus it is not $\Sigma$-cyclic) with $G / p^{\omega} G \cong A$. Applying Theorem 1.3 of $[\mathbf{7}], G$ does not has a nice basis (see also Proposition 4.5 below).

Example 4.3. In Proposition 4.1, the niceness of $N$ in $G$ is also necessary.

Let $G$ be a reduced group without a nice basis with a countable basic subgroup $N$ (for example, if $G / p^{\omega} G$ is torsion-complete with a countable basic subgroup and $p^{\omega} G$ is not $\Sigma$-cyclic, it follows from [7] that $G$ does not have a nice basis). Clearly, $N(\omega)=\{0\}$ is satisfied and $A=G / N$ is divisible, hence it does have a nice basis owing to [2].

We now investigate the hypothesis that $N(\omega)=\{0\}$ in the last result by contrasting it with the case where $G$ is an $\omega$ - $\aleph_{0}$-elongation of $A$ by $N$; i.e., we are contrasting the situation in which $N \cap p^{\omega} G=\{0\}$ with the situation in which $N=p^{\omega} G$. We begin by considering an apparently unrelated notion.

A separable valuated vector space $V$ (i.e., $V(\omega)=\{0\}$ ) will be called essentially finitely indecomposable (or efi for short) if (1) it is unbounded 
(in the sense that $V(m) \neq\{0\}$ for all $m<\omega$ ); and (2) there is no valuated decomposition $V=W \oplus F$ where $F$ is an unbounded free valuated vector space. For example, if $V$ is complete in the $p$-adic topology (i.e., if it is isometric to the socle of a torsion-complete group), then $V$ is efi. The following topological characterization utilizes ideas from [7].

Proposition 4.4. An unbounded separable valuated vector space $V$ is efi iff $V$ cannot be expressed as the ascending union of a sequence of closed subspaces $C_{0} \subseteq C_{1} \subseteq C_{2} \subseteq \cdots$ such that $V / C_{m}$ is unbounded for every $m<\omega$.

Proof: If there is a decomposition $V=W \oplus F$ and we let $F=\oplus_{i<\omega} B_{i}$ where $B_{i}(i) \neq\{0\}$, then we may simply let $C_{m}=W \oplus\left(\oplus_{i<m} B_{i}\right)$. Therefore, $V / C_{m} \cong \oplus_{m \leq i} B_{i}$, so that $C_{m}$ is closed and $V / C_{m}$ is unbounded.

Conversely, if we have the collection of closed subgroup $C_{m}$ satisfying the above, then let $x_{m} \in V-C_{m}$ be chosen so that $v\left(x_{m}\right) \geq m$ and $\left\langle x_{m}\right\rangle$ is a valuated summand of $V / C_{m}$; denote the valuated projection $V / C_{m} \rightarrow\left\langle x_{m}\right\rangle$ by $\pi_{m}$. If $f: V \rightarrow \prod_{m<\omega}\left\langle x_{m}\right\rangle$ is given by $f(x)=\left(\pi_{m}(x+\right.$ $\left.\left.C_{m}\right)\right)_{m<\omega}$, then it is easy to verify that $f$ is a valuated homomorphism whose image is unbounded and is actually contained in the free valuated vector space $\oplus_{m<\omega}\left\langle x_{m}\right\rangle$. If we let $W$ be the kernel of $f$, then $V=W \oplus F$, where $F$ is an unbounded free valuated vector space.

Proposition 4.5. If $A$ is any unbounded separable group that has a subsocle $P \subseteq A[p]$ which is efi, then there is a group $G$ which is an $\omega$-\$ $\aleph_{0}$-elongation of $A$, such that $G$ does not have a nice basis.

Proof: Let $N$ be any reduced countable group which is not $\Sigma$-cyclic, i.e., $p^{\omega} N \neq\{0\}$. Let $D$ be a dense subspace of $A[p]$ such that $A[p] / D$ is countably infinite and $D+P(i)=A[p]$ for all $i<\omega$ [start with a dense subspace $D^{\prime}$ of $P$ such that $P / D^{\prime}$ is countably infinite, and if $W$ is a subspace of $A[p]$ such that $P \oplus W=A[p]$, then we can let $\left.D=D^{\prime}+W\right]$.

By Lemma 3.2 there is a group $G$ which is an $\omega$-elongation of $A$ by $N$ such that $D=\left[G[p]+p^{\omega} G\right] / p^{\omega} G \subseteq A[p]$. Suppose now that $G$ actually does have a nice basis $M_{0} \subseteq M_{1} \subseteq M_{2} \subseteq \cdots$. If for each $i<\omega$ we let $L_{i}=\left[M_{i}+N\right] / N$, then $L_{i}$ is closed in the $p$-adic topology on $A$ and their union is all of $A$. It follows that $P$ is the union of the closed subspaces $C_{i}=P \cap L_{i}$. Since $P$ if efi, it follows from Proposition 4.4 that, for some $i, P(i) \subseteq P \cap C_{i} \subseteq L_{i}$. We therefore have $A[p] \subseteq D+L_{i}$.

If $x \in N$, then find $y \in G$ such that $p y=x$. Since $y+N \in A[p]$ there is a $w \in G[p]$ and $z \in M_{i}$ such that $y+N=(w+z)+N$. So there is a $u \in N$ such that $y=w+z+u$. This implies that $x=p y=$ $p w+p z+p u=p z+p u$. Note that $p z \in M_{i}(\omega), p u \in p N$, so that we 
can conclude $N=M_{i}(\omega)+p N$. This, in turn, implies that $N / M_{i}(\omega)$ is divisible. Since $M_{i}$ is nice in $G, M_{i}(\omega)$ is nice in $N$. Since $N$ is reduced and $N / M_{i}(\omega)$ is divisible, we must have that $N=M_{i}(\omega) \subseteq M_{i}$. This is a contradiction, because $M_{i}$ being a part of a nice basis is $\Sigma$-cyclic, though we assumed $N$ was not.

Example 4.6. There are $p^{\omega+1}$-projective groups $A$ satisfying Proposition 4.5 .

If $P$ is any separable valuated vector space which is efi, then there is a separable group $A$ containing $P$ such that the valuation on $P$ agrees with the height function on $A$ and $A / P$ is $\Sigma$-cyclic.

Remark 2. If we start with the group $A$ from Example 4.6 and construct the group $G$ as in Proposition 4.5, then since $G$ does not have a nice basis, it follows from [2] that it is not $p^{\omega+1}$-projective. Therefore, in Theorem 4.2 of $[\mathbf{6}]$, the condition of separability is necessary and cannot be eliminated.

Following [7], the separable group $A$ is said to have the nice basis extension property if for all $\omega$-elongations $G$ of $A$, if $p^{\omega} G$ has a nice basis, then so does $G$. By Corollary 2.13 of [7], the Continuum Hypothesis $(\mathrm{CH})$ implies that when $A$ has a countable basic subgroup, then $A$ has the nice basis extension property iff it is $\Sigma$-cyclic, and it was asked whether all groups with this property are necessarily $\Sigma$-cyclic. The following is related to this question by restricting to the case of $\omega$ - $\aleph_{0}$-elongations.

Example 4.7. In the presence of $\mathrm{MA}+\neg \mathrm{CH}$, there is a separable group $A$ which is not $\Sigma$-cyclic, with the property that every $\omega$ - $\aleph_{0}$-elongation $G$ of $A$ has a nice basis. This group may be chosen to be $p^{\omega+1}$-projective.

Let $A$ be as in Theorem 3.4(a). If $G$ is an $\omega$-elongation of $A$, then since $A$ has the $\omega$-cps-elongation property, $G \cong C \oplus S$, where $C$ is countable and $S$ is separable. Since $C$ is countable, it is totally projective, and hence it has a nice basis by [2]. Since $S$ is separable, it clearly has a nice basis, as well. Therefore, in view of $[\mathbf{2}], G$ has a nice basis, as required. The last part follows from Theorem 3.3.

The following completely answers Problem 4 of [7].

Example 4.8. There are groups $G_{1}$ and $G_{2}$ with $p^{\omega} G_{1} \cong p^{\omega} G_{2}$ and $G_{1} / p^{\omega} G_{1} \cong G_{2} / p^{\omega} G_{2}$ such that $G_{1}$ has a nice basis but $G_{2}$ does not have a nice basis. 
Suppose $A^{\prime}$ is a separable group with a subsocle $P$ which is efi, and $B$ is an unbounded $\Sigma$-cyclic group. So if $A=A^{\prime} \oplus B$, then since $P$ is also a subsocle of $A$, by Proposition 4.5 , there is an $\omega$ - $\aleph_{0}$-elongation $G_{2}$ of $A$ by a countable group $N=p^{\omega} G_{2}$ which does not have a nice basis. Since $N$ is countable and $B$ is unbounded, there a dsc-group $H$ such that $p^{\omega} H \cong N$ and $H / p^{\omega} H \cong B$. If we let $G_{1}=H \oplus A^{\prime}$, then $H$ has a nice basis by $[\mathbf{2}]$ (since it is a dsc-group and hence totally projective), $A^{\prime}$ has a nice basis (since it is separable), so that $G_{1}$ has a nice basis in virtue of $[\mathbf{2}]$, as required.

Question 1. Is the converse of Proposition 3.1(b) valid in ZFC? That is, if $A$ is a separable group with the $\omega$-cps-elongation property, does it follow in ZFC that $A$ has the cps-elongation property?

Question 2. Suppose $A$ is a separable group with the property that every $\omega$-§ $\aleph_{0}$-elongation $G$ of $A$ has a nice basis. In $V=L$, does it follow that $A$ must be $\Sigma$-cyclic? (Note that by Example 4.7, this does not hold in a model of MA $+\neg \mathrm{CH}$.)

Question 3. Suppose $A$ is a separable group with the property that every $\omega$-elongation $G$ by a totally projective group $X=p^{\omega} G$ has a nice basis. Can we conclude that $A$ is $\Sigma$-cyclic? (If this holds, then all such $G$ will also be totally projective.)

\section{Almost totally projective groups}

A group $G$ is almost totally projective if it has a collection of nice subgroups $\mathcal{N}$ with the properties:

(0) $\{0\} \in \mathcal{N}$.

(1) If $\left\{H_{i}\right\}_{i \in I}$ is an ascending chain of subgroups in $\mathcal{N}$, then $\cup_{i \in I} H_{i} \in$ $\mathcal{N}$.

(2) If $C \subseteq G$ is countable, then there is a countable $M \in \mathcal{N}$ such that $C \subseteq M$.

We pause to review the following.

Proposition 5.1 ([15], [5]). If $K$ is a subgroup of the reduced group $H$ such that $H / K$ is countable and $K$ is almost totally projective, then $H$ is almost totally projective.

Proposition 5.2 ([5]). If $K$ is a countable and nice subgroup of the reduced group $H$ such that $H / K$ is almost totally projective, then $H$ is almost totally projective. 
More directly, we can state the following (see $[\mathbf{6}]$ for totally projective groups).

Proposition 5.3. Suppose $A$ is reduced and $G$ is an $\aleph_{0}$-elongation of $A$ by $X$. If $G$ is almost totally projective, then $A$ is almost totally projective.

Proof: Let $\mathcal{N}$ satisfy (0), (1), (2) above, and let $\mathcal{N}^{\prime}=\{0\} \cup\{N / X$ : $N \in \mathcal{N}$ and $X \subseteq N\}$. First, note that if $N / X \in \mathcal{N}^{\prime}$, then $N$ is nice in $G$, so that $N / X$ is nice in $A$. Next, that $\mathcal{N}^{\prime}$ satisfies (0) is immediate. As for (1), if $\left\{H_{i} / X\right\}_{i \in I}$ is an ascending chain of subgroups in $\mathcal{N}^{\prime}$, then $\left\{H_{i}\right\}_{i \in I}$ is an ascending chain of subgroups in $\mathcal{N}$. It follows that $\cup_{i \in I} H_{i} \in \mathcal{N}$, so that $\cup_{i \in I}\left(H_{i} / X\right) \in \mathcal{N}^{\prime}$, proving that (1) holds for $\mathcal{N}^{\prime}$. Finally, if $C / X$ is countable for some $C \leq G$, then so is $C$ and hence there is a countable $M \in \mathcal{N}$ such that $C \subseteq M$. Thus $C / X \subseteq M / X \in \mathcal{N}^{\prime}$, whence (2) follows as required.

Example 5.4. The converse of Proposition 5.3 need not hold.

By Example 2.3 of [6], there is a separable non $\Sigma$-cyclic group $G$ with a countable but not nice subgroup $X$ such that $A=G / X$ is a dsc-group, hence is almost totally projective, with $p^{\omega} A$ uncountable. If this $G$ were, in fact, almost totally projective, then $X$ would be contained in a countable closed subgroup $N \in \mathcal{N}$. If $\bar{X}$ is the $p$-adic closure of $X$ in $G$, then $p^{\omega} A=\bar{X} / X \subseteq N / X$ would be countable, contrary to hypothesis.

We have the following consequence of the above.

Corollary 5.5. Let $G$ be a reduced group which is a nice- $\aleph_{0}$-elongation of $A$. Then $G$ is almost totally projective iff $A$ is almost totally projective.

Proof: Sufficiency follows from Proposition 5.2 and necessity follows from Proposition 5.3.

Observe that similar results were proved by Dieudonné $[\mathbf{9}]$ for $\Sigma$-cyclic groups and by Balof-Keef [1] for almost $\Sigma$-cyclic groups.

We conclude with the following result.

Theorem 5.6. Suppose $G$ and $A$ are reduced groups and $f: G \rightarrow A$ is an $\omega_{1}$-bijective homomorphism. If $G$ is almost totally projective, then $A$ is almost totally projective.

Proof: Let $I$ be the image of $f$ and $X$ be the kernel of $f$. Note that $G$ is an $\aleph_{0}$-elongation of $I$ by $X$, so by Proposition 5.3, $I$ is almost totally projective. Since $A / I$ is also countable, by Proposition 5.1, we can conclude that $A$ is almost totally projective, as required. 
The above discussion leads naturally to the following.

Question 4. If $f: G \rightarrow A$ is an $\omega_{1}$-bijective homomorphism and $A$ is totally projective, what we can say about $G$ ?

\section{References}

[1] B. BAlof AND P. KeEF, Invariants on primary abelian groups and a problem of Nunke, Note Mat. (to appear).

[2] P. V. DANChev, Nice bases for primary abelian groups, Ann. Univ. Ferrara Sez. VII Sci. Mat. 53(1) (2007), 39-50.

[3] P. V. DANCheV, On countable extensions of primary abelian groups, Arch. Math. (Brno) 43(1) (2007), 61-66.

[4] P. V. Danchev, Generalized Dieudonné and Hill criteria, Port. Math. 65(1) (2008), 121-142.

[5] P. V. DANChev, On extensions of primary almost totally projective abelian groups, Math. Bohem. 133(2) (2008), 149-155.

[6] P. V. DancheV and P. W. Keef, Generalized Wallace theorems, Math. Scand. 104(1) (2009), 33-50.

[7] P. V. Danchev and P. W. Keef, Nice bases and thickness in primary abelian groups, Rocky Mountain J. Math. (to appear).

[8] P. V. Danchev and P. W. Keef, $n$-Summable valuated $p^{n}$-socles and primary abelian groups, Comm. Algebra (to appear).

[9] J. Dieudonné, Sur les p-groupes abéliens infinis, Port. Math. 11 (1952), 1-5.

[10] P. C. Eklof And A. H. Mekler, "Almost free modules. Settheoretic methods", Revised edition, North-Holland Mathematical Library 65, North-Holland Publishing Co., Amsterdam, 2002.

[11] L. Fuchs, "Infinite abelian groups", Vol. I, Pure and Applied Mathematics 36, Academic Press, New York-London, 1970; "Infinite abelian groups", Vol. II, Pure and Applied Mathematics 36-II, Academic Press, New York-London, 1973.

[12] L. Fuchs, Vector spaces with valuations, J. Algebra 35 (1975), $23-38$.

[13] L. Fuchs, On $p^{\omega+n}$-projective abelian $p$-groups, Publ. Math. Debrecen 23(3-4) (1976), 309-313.

[14] P. A. GRIFFith, "Infinite abelian group theory", The University of Chicago Press, Chicago, Ill.-London, 1970.

[15] P. Hill and W. Ullery, Isotype separable subgroups of totally projective groups, in: "Abelian groups and modules" (Padova, 1994), Math. Appl. 343, Kluwer Acad. Publ., Dordrecht, 1995, pp. 291-300. 
[16] J. IRWin And P. KeEF, Primary abelian groups and direct sums of cyclics, J. Algebra 159(2) (1993), 387-399.

[17] P. KeEF, On iterated torsion products of abelian p-groups, Rocky Mountain J. Math. 21(3) (1991), 1035-1055.

[18] C. Megibben, Crawley's problem on the unique $\omega$-elongation of p-groups is undecidable, Pacific J. Math. 107(1) (1983), 205-212.

[19] C. Megibien, $\omega_{1}$-separable p-groups, in: "Abelian group theory" (Oberwolfach, 1985), Gordon and Breach, New York, 1987, pp. $117-136$.

[20] A. H. Mekler ANd S. Shelah, $\omega$-elongations and Crawley's problem, Pacific J. Math. 121(1) (1986), 121-132.

[21] R. J. Nunke, Purity and subfunctors of the identity, in: "Topics in Abelian Groups" (Proc. Sympos., New Mexico State Univ., 1962), Scott, Foresman and Co., Chicago, Ill., 1963, pp. 121-171.

[22] F. Richman and E. A. Walker, Valuated groups, J. Algebra 56(1) (1979), 145-167.

Peter V. Danchev:

Department of Mathematics

Plovdiv University "Paisii Hilendarski"

Plovdiv 4000

Bulgaria

E-mail address: pvdanchev@yahoo.com

Patrick W. Keef:

Department of Mathematics

Whitman College

Walla Walla, WA 99362

USA

E-mail address: keef@whitman.edu

Primera versió rebuda el 24 de març de 2009, darrera versió rebuda el 21 de setembre de 2009. 